\title{
A Case Study in Branch Testing Automation
}

\author{
A. Bertolino* ${ }^{*}$ R. Mirandola ${ }^{\circ}$, E. Peciola ${ }^{\dagger}$ \\ * Istituto di Elaborazione della Informazione del CNR, Pisa, Italy. \\ Laboratory for Computer Science, Università di Roma "Tor \\ Vergata", Italy. \\ † Ericsson Telecomunicazioni S.P.A., Roma, Italy.
}

\begin{abstract}
We present a real world experience with some recent research results aimed at improving the branch testing process. The results considered consist into (i) a method for the automatic derivation of sets of paths that satisfy the branch testing criterion and into (ii) a bound on the number of test paths needed for achieving $100 \%$ branch coverage. The derivation of test path sets (point $i$ ) is based on a new method for the static analysis of the program flowgraph; the method has been implemented within a prototype tool, called BAT. The computation of the bound (point ii), called $\beta$ branch, considers those control flow paths with a low number of predicates, that are more likely feasible. BAT and $\beta$ branch, have been validated on object oriented $\mathrm{C}++$ software, developed to control a new generation of telecommunications systems, within the test environment of Ericsson Telecomunicazioni. The case study regarded the basic test phase, that is the first step of the testing process and is appropriate for the application of the proposed theory, since it includes the branch testing of program units up to a prescribed coverage measure. While the experimentation is still on-going, we describe some preliminary results.
\end{abstract}

\section{Keywords}

Branch coverage, ddgraph, infeasible path, statistical test, test path, unconstrained branches.

\section{INTRODUCTION}

Software testing (Beizer, 1990) consists in the validation of computer programs through the observation of a meaningful sample of executions chosen from the potentially infinite execution domain.

To select an adequate set of test cases, different strategies can be followed, based either on program specification or on program structure. Whatever strategy is selected, measures of structural coverage (Rapps and Weyuker, 1985) can be used to determine how thorough the executed test cases have been. In particular, branch coverage, which requires that each branch alternative in a program is exercised at least once, is commonly accepted as a "minimum. mandatory testing requirement" (Beizer, 1990). 
The branch testing process involves:

i) selecting a set of test cases trying to exercise every (as yet uncovered) program branch;

ii) executing the program on the selected test cases and monitoring the branches actually exercised;

iii) evaluating the ratio between the number of executed branches and the total number of branches in the program. If this ratio reaches a predefined threshold, the test is stopped; otherwise, more test cases must be devised: the process is repeated from step i).

In this procedure, steps ii) and iii) can be mechanised, and in fact several dynamic coverage analysers are today available that instrument the program and then can probe the exercised branches as the tests are executed. The first step, that clearly involves the largest part of the test effort, is instead left to the tester's skill and creativity.

In (Bertolino and Marré, 1994), a method has been proposed that can help the tester in this task. The method derives a set of test paths that will cover every arc in the program flowgraph. Then, the task of the tester to accomplish step i) would be "reduced" to that of finding a set of test inputs that execute the suggested paths. Since the paths are statically generated, they might inevitably include infeasible paths, i.e., control flow paths that are exercised by no input data. However, to reduce the incidence of this problem, the proposed method generates "simple" paths, i.e., paths that involve a low number of predicates. Indeed, the shorter a path is, the more likely it is that such path is feasible: this is not only intuitively true, but has also been demonstrated statistically (Yates and Malevris, 1988).

The algorithm exploited in the generation of the test paths has been shown to be correct and efficient in theory (Bertolino and Marré, 94). However, its practical usefulness has yet to be confirmed. In principle, the tester task should be made easier by having at disposal the "right" set of paths. In practice, the parameters involved in a real world test process are so many and so complex that only an empirical validation of the method can be trusted upon. In this paper we describe a real world case study with the test path generation method and illustrate some preliminary results.

The described case study involved another related research result, the $\beta$ branch, bound (Bertolino and Marré, 95). Testing activities consume a considerable fraction of the time and resources spent to produce a software product; therefore it would be useful to have a way to estimate this testing effort. Indeed, knowing in advance how much effort will be needed to test a given program is essential to the manager to plan the software process.

Now, control flow analysis can be used also to predict the number of test cases needed to guarantee a particular structural coverage. In particular, the number of test paths needed to achieve branch coverage can be regarded as a measure of the minimum mandatory effort to test a given program, regardless of the particular strategy used in the selection of test data. So, it would be useful to be able to compute a lower bound on the number of test cases needed to achieve branch coverage. This number does not correspond to the theoretical minimum number of paths needed to cover the program flowgraph: to minimise the number of paths, in fact very long and complicated paths should be considered. On the contrary, in the computation of this bound those paths containing a low number of decisions should be considered, that are more likely feasible: each path should not enter different loops and, in case a path enters a loop, then it will be iterated just once.

Following this criterion, in (Bertolino and Marré, 1995) a number, called the $\beta$ branch, bound, has been proposed as a metric to predict the number of test cases needed to branch test a given program. In the case study described in this paper, we have validated the performance of $\beta$ branch on a number of program units that have been tested according to the branch testing strategy and for which the real number of executed test cases has been collected. Besides, since the cyclomatic number (McCabe, 1976) of the program flowgraph is often used as such a bound, we have performed the same analysis on this number for comparison.

In Section 2, we provide some theoretical background. In Section 3, the real world test environment used in the case study, that is that of Ericsson Telecomunicazioni, is presented. In Section 4, the experience and the results obtained up to this point are described. Finally, in Section 5 some conclusions and future developments are briefly sketched. 


\section{THEORETICAL BACKGROUND}

In this section we provide a short background useful to understand a little more about the path generation method and the $\beta$ branch bound, which were the subjects of this case study. More extensive material can be found in (Bertolino and Marré, 1994) and (Bertolino and Marré, 1995), respectively.

\subsection{Unconstrained arcs}

A program structure is conveniently analysed by means of a directed graph, called flowgraph, that gives a graphical representation of the program control flow. A program control flow may be mapped onto a flowgraph in different ways. In our approach, we use a flowgraph representation called ddgraph, which is particularly suitable for the purposes of branch testing. Each arc in a ddgraph directly corresponds to a program branch; thus, program branch coverage is immediately measured in terms of ddgraph arc coverage.

The following is our definition of ddgraphs.

\section{Definition 1: Ddgraph}

A ddgraph is a digraph $G=(V, E)$ with two distinguished arcs $e_{0}$ and $e_{k}$ (which are the unique entry arc and exit arc, respectively), such that any other $\operatorname{arc}$ in $G$ is reached by $e_{0}$ and reaches $e_{k}$, and such that for each node $n$ in $V, n \neq \mathrm{T}\left(e_{0}\right), n \neq \mathrm{H}\left(e_{k}\right)$, (indegree $(n)+$ outdegree $(n))>2$, while indegree $\left(\mathrm{T}\left(e_{0}\right)\right)=0$ and outdegree $\left(\mathrm{T}\left(e_{0}\right)\right)=1$, indegree $\left(\mathrm{H}\left(e_{k}\right)\right)=1$ and outdegree $\left(\mathrm{H}\left(e_{k}\right)\right)=0$.

Ddgraph arcs are associated to program branches. A branch is here defined as a strictly sequential set of program statements. Ddgraph's nodes may correspond to a branching or to a joining in the program control flow (which may be empty, i.e., not associated to any piece of code). An example of a ddgraph is shown in the upside window of figure 1.

We exploit the dominance relationship (Hecht, 1977) from the graph theory, which impose a partial ordering on the nodes of a digraph. By applying dominance, and its symmetric relationship of implication (elsewhere known as post-dominance), to the arcs of a ddgraph, we obtain two trees (whose nodes represent the ddgraph arcs), the Dominator Tree $(D T(G))$ and the Implied Tree $(I T(G))$, rooted at $e_{0}$ and at $e_{k}$, respectively. The $D T(G)$ and $I T(G)$ for the ddgraph given in figure 1 are shown in figure 2.

Dominance and implication allow us to identify a subset of ddgraph arcs that is very useful for branch testing: the set of unconstrained arcs (Bertolino, 1993). The fundamental property of unconstrained arcs is that a path set that covers all the unconstrained arcs of a ddgraph also covers all the arcs in the ddgraph; besides, the unconstrained arcs form the minimum set of arcs with that property. This property has been proved in (Bertolino, 1993).

We can immediately find the set of unconstrained arcs by using the dominator tree and the implied tree of a ddgraph $G$. We can obtain the set $U E(G)$ of the unconstrained arcs of $G$ as $D T L(G) \cap I T L(G)$, where $D T L(G)$ is the set of leaves of $D T(G)$ and $I T L(G)$ is the set of leaves of $I T(G)$. This can be seen in figure 2, where the unconstrained arcs for the ddgraph of figure 1 are those that are double-circled.

\subsection{Generation of branch covering set of paths}

A set of paths such that each branch in a program is covered by at least one path in the set is called a branch covering set of paths. On the ddgraph $G$ of that program, the branch covering set of paths will correspond to a set of paths that covers every arc of $G$ (note that, due to the infeasibility problem, the reverse is not necessarily true).

The algorithm FTPS (for FIND-A-TEST-PATH-SET) is a recursive algorithm that uses $D T(G)$ and $I T(G)$ to construct a set $\pi=\left\{P_{1}, \ldots, P_{n}\right\}$ of paths that covers every arc of a given 


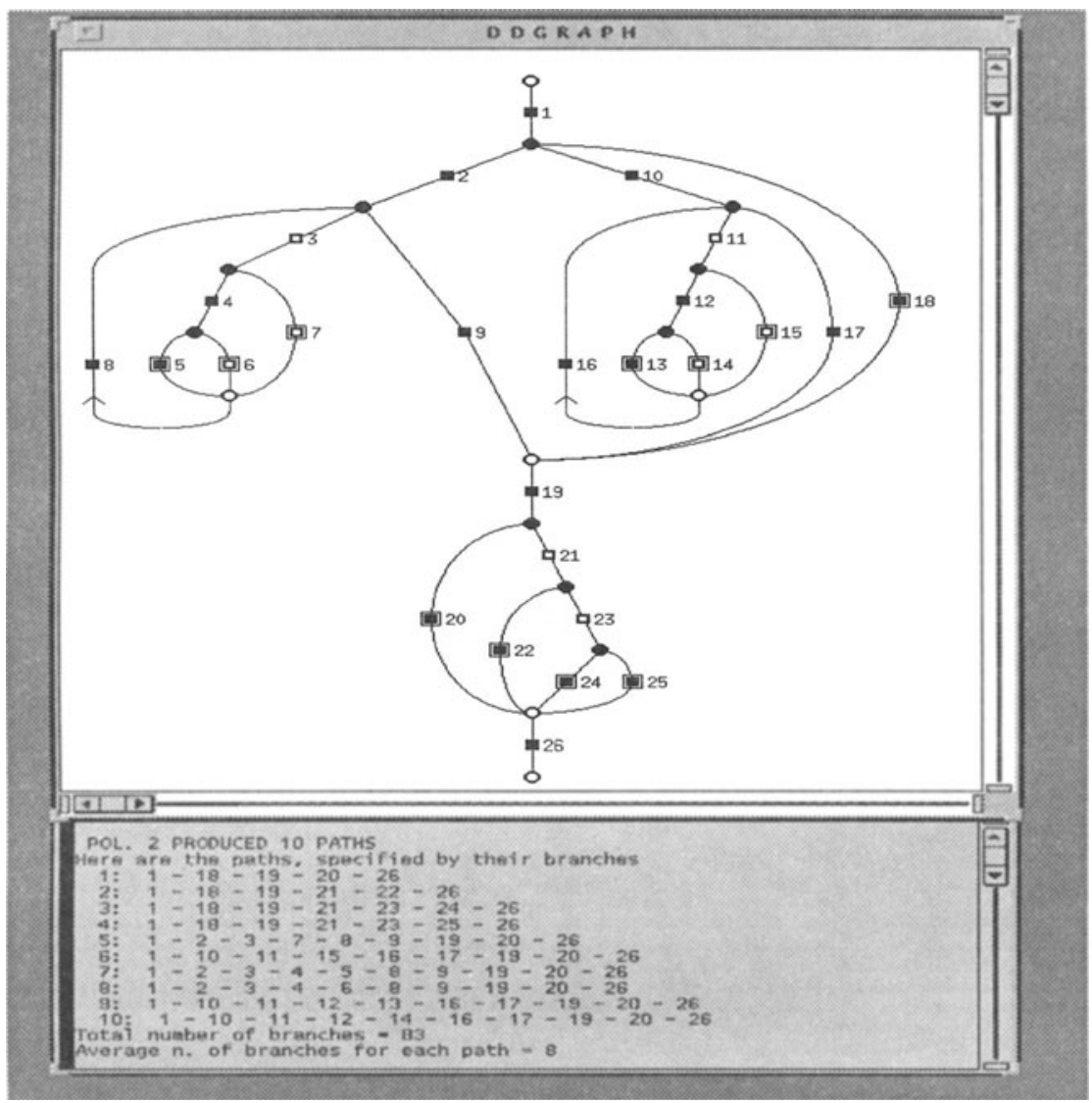

Figure 1 An example of a ddgraph.

ddgraph $G$. To do this, FTPS constructs a set of paths that covers all the unconstrained arcs of the ddgraph: the fundamental property of unconstrained arcs then guarantees that the path set found covers every arc.

Algorithm FTPS builds the set $\pi$ of paths one path at a time. To construct each path, FTPS selects an as yet uncovered unconstrained arc $e_{u}$ and then calls a recursive function FIND-APATH. This function constructs a path from $e_{0}$ to $e_{k}$, using arc $e_{u}$, simply by concatenating the unique path $P_{D T}$ in $D T(G)$ from the root $e_{0}$ to the leaf $e_{u}$, with the unique path $P_{I T}$ in $I T(G)$ from the leaf $e_{u}$ to the root $e_{k}$.

The recursion is necessary since the sequence of arcs obtained by concatenating $P_{D T}$ with $P I T$ might not be a proper path in $G$, because it might contain not adjacent arcs. Whenever two arcs $e_{i}$ and $e_{j}$ in the derived path are not adjacent arcs in $G$, the algorithm makes a recursive call to FIND-A-PATH and derives a path that joins the two arcs on an appropriately 


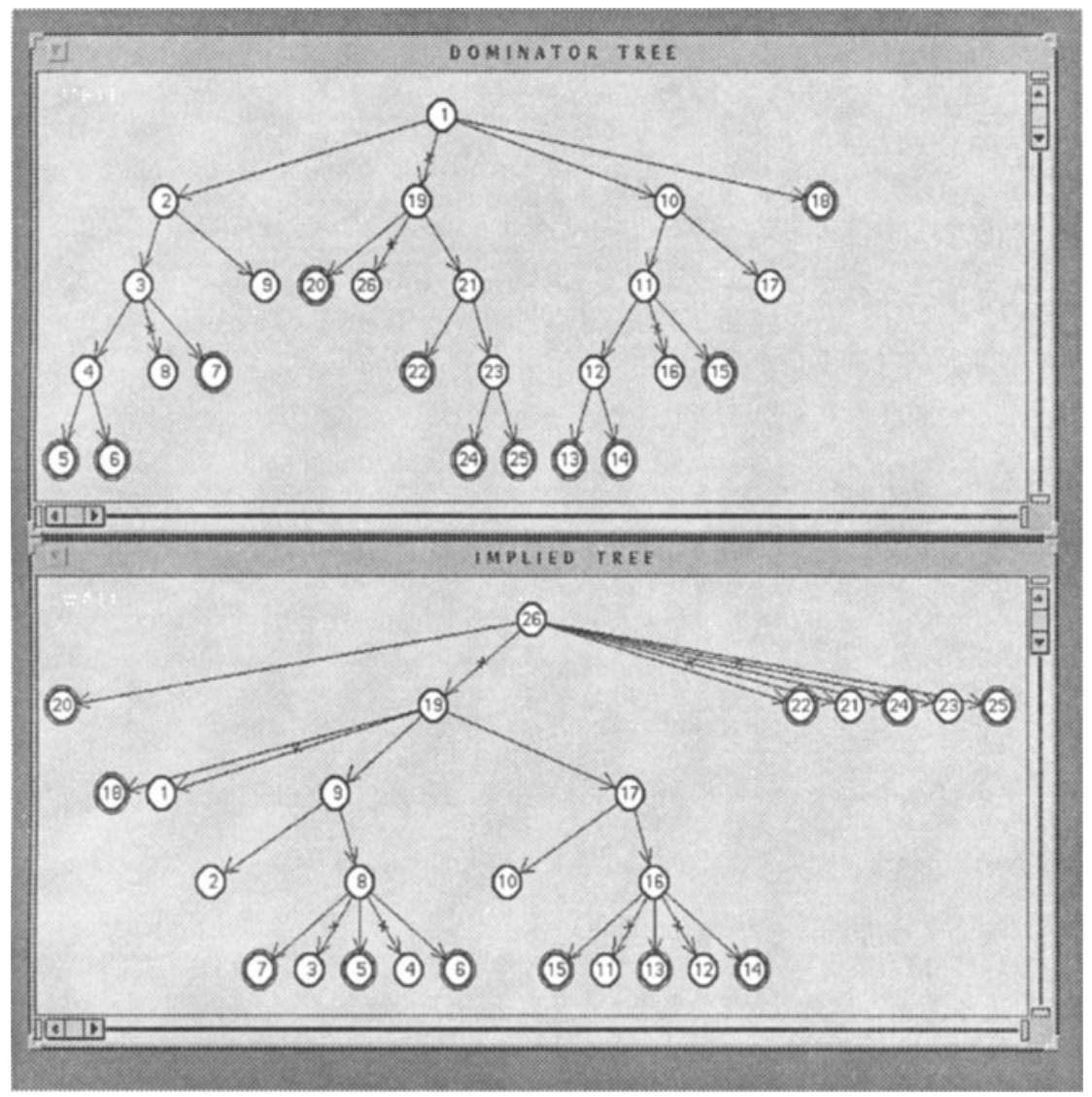

Figure 2 DT(G) and IT(G) for the ddgraph of figure 1.

derived sub-ddgraph. At each recursive iteration, another unconstrained arc $\boldsymbol{e}^{\prime} \boldsymbol{u}$ must be selected: to reduce the generation of infeasible paths, the selection is made so that the resulting path will contain a low number of predicates. The paths found by the function FIND-A-PATH in this way may contain cycles (whenever an unconstrained arc within a cycle is selected) but, by construction, each cycle within each path will be iterated at most once.

Algorithm FTPS is implemented within the prototype tool BAT. Figure 1 and figure 2 are both printouts from BAT screens; in particular, in the bottom window of figure 1 the paths suggested by the tool BAT for branch testing the ddgraph in the same figure can be seen.

\subsection{The Bbranch bound}

The $\beta$ branch bound provides a "meaningful lower bound" to the cardinality of a set of paths that is useful to guarantee branch coverage. 
Let us first describe our approach intuitively: given a ddgraph $G=(V, E)$, we have seen that there exists a minimum set of arcs, the set $U E$ of unconstrained arcs, which guarantees the coverage of every arc in $G$. Then, of course, the number of test cases needed to achieve branch coverage is $\leq|U E|$, where $|S|$ denotes the number of elements in a set $S$. However, for an arbitrary ddgraph, an entry-exit path may, in general, cover more than one unconstrained arc. Depending on how unconstrained arcs are combined together into one path, different sets of test paths can be obtained, with different cardinalities.

To combine unconstrained arcs so as to obtain meaningful paths, we introduce the relation of weak incomparability between arcs (Bertolino and Marré, 1995). Intuitively, two arcs are weakly incomparable if:

- either of them can be covered by a simple path, but they cannot be both covered by the same simple path, or

- one of them can be covered by a simple path, but the other cannot, or

- they belong to the same cycle and one reaches the other only by entering the cycle at least twice, or

- they belong to different cycles.

A meaningful path is thus a path that does not contain two weakly incomparable unconstrained arcs.

Obviously, for a ddgraph that does not contain cycles, two arcs are weakly incomparable if and only if they are incomparable.

Combining the notions of unconstrained arcs and of weakly incomparable arcs, $\beta$ branch can be defined as the maximum number of weakly incomparable unconstrained arcs in a ddgraph. Denoting by $L W I(U E)$ the largest set of weakly incomparable unconstrained arcs in $G$, we can define $\beta$ branch as follows:

$$
\beta_{\text {branch }}=|L W I(U E)| \text {. }
$$

\section{THE ERICSSON TEST ENVIRONMENT}

The Software Department of Ericsson Telecomunicazioni R\&D decided about one year ago to start investments in research activity dealing with software testing, after having analysed the costs of the test phase of some projects developed using advanced technology, with iterative processes and incremental development. Considering a complete development process consisting of analysis, design, implementation and basic test, it was evaluated that the cost of basic test activity can be up to $2 / 3$ of the total development cost (average cost of basic test of a method is inversely proportional to its amount of new or changed lines of code).

In addition, in projects with complicated test strategies and dependencies between different phases, it is a basic goal to pass milestones on schedule. The $\beta$ branch metric, related to the number of test paths needed to achieve $100 \%$ branch coverage, could have been a good support in the detailed estimation of expected effort of a basic test activity.

\subsection{Project and methodology description}

The project chosen for the case study is carried out by Ericsson Telecomunicazioni R\&D in cooperation with the Headquarters in Stockholm and other Ericsson subsidiaries. It was one of the biggest projects developed in Europe using Object Oriented Technology and the first experience for Ericsson Telecomunicazioni. The project started in 1992, after a prototyping phase, and continued in overlapping development phases, with further functionalities added at each incremental step. Milestones were used to coordinate phases and steps.

The application developed in Rome consists of software controlling a synchronous triplicated switch matrix that is the core of a Digital Cross Connect System used for the new generation Transmission Network based upon the SDH technology. The software is designed following Object Oriented methodology and implemented in the C++ language using the SUN OS platform for both development and target environments. 
A specific development process consisting of Analysis, Design, Implementation and Test, based on the ObjectOry methodology (Jacobson, 1992), was implemented for this project.

Due to the tough release plan from the customer, the software modelled at early phase could not be restructured and software units grew in size and complexity.

The real world testing process chosen for the case study is one step of the incremental development of this project. Analysis, design, implementation and test were performed.

\subsection{Basic Test strategy}

The test strategy for this project included four different test phases all of them supported by the methodology:

- Basic Test, testing the smallest module (test object) in the system. The goal is to verify design specification;

- Integration Test, testing a functional area. All modules involved in that area are integrated;

- Function Test, verifying system functions (use cases);

- System Test, verifying system performance and architectural requirements.

The basic test activity, which is the phase monitored in our case study, is a quite essential part of the testing phase: a poor basic test can never be compensated by other test activities.

The goal of basic test is to check the code against coding rules, design rules and design specification reaching a branch coverage greater then $80 \%$. This minimum coverage range is a customer requirement. The TCAT tool (STW, 1991) is used to measure it.

The procedure used is the following: a test program is written; for each test case in the test program, input data are provided and produced output data are controlled against the expected results. $\log$ files are produced in which the result of each test is registered. Stubs are used to simulate test object environment.

\section{DESCRIPTION OF THE CASE STUDY}

The objective of the case study was twofold:

1) to evaluate BAT effectiveness as a tool to improve the branch testing process. In particular, we are interested to validate the two specific aspects:

1.a) providing the tester with a set of paths that guarantees $100 \%$ branch coverage can reduce the test effort;

1.b) the paths derived by BAT are very likely feasible;

2) to evaluate the $\beta$ branch bound as a metric for the prediction of the test effort needed to obtain branch coverage.

Accordingly the case study has been conducted in two separate phases, that are described in the two following sections.

\subsection{Evaluation of BAT effectiveness}

In the planning of the case study, we found that the evaluation of BAT effectiveness is made difficult by the following two problems:

i) to be able to compare the testing with and without BAT, we would need to run two independent testing sessions on the same set of program units. However, if one same person is used to run both sessions, with and without BAT, of course the second session would benefit from the experience gained in the previous session, whichever of the cases is taken first. On the other side, if we use two different persons to run the two testing sessions separately, the experiment would be affected by the possible discrepancy between each tester's skill. 
ii) Even admitting that we can overcome the above difficulty, for instance finding two perfectly equivalent testers, we are anyway faced with the problem that the program units that were tested are part of a real project, and so testing resources and schedule are constrained. This second difficulty resolved definitely (and negatively) the doubt whether the test of each program could be duplicated.

Before starting the testing phase, the biggest and most complex modules were selected to validate the BAT tool on them. Some adaptations were required to improve BAT performance.

Common feeling of all participants in this stage was that the tool could have been useful to fulfil the coverage requirement fixed by the customer, but if used after a first phase in which black box tests are run, derived from design specification.

Following this, the test strategy was defined. An expert tester and a beginner were chosen for the case study. Neither of them had performed coding of the modules they were going to test.

It was decided to execute the test phase according to the strategy described below:

- perform basic test in the traditional way based on test specification;

- evaluate test coverage;

- split randomly the set of program units that have not fulfilled the test coverage requirements into two groups: the STANDARD group, to be tested following the standard internal procedures, i.e., without the support of the BAT tool, and the BAT group, to be tested using the BAT tool. In particular, for the STANDARD group the tester tried manually to guess additional tests that would raise the coverage. For the BAT procedures, the tester tried to exercise the paths suggested by the tool. In both cases, the test is stopped when a given threshold of the average coverage for all the program units in a module is reached.

Modules to be tested were selected according to project priority at that time. The expert tester was planned to test two modules, the beginner performed the test of just one module. To avoid distortion of results, program units from the two groups had been alternatively tested, i.e., one from the STANDARD group and then one from the BAT group .

Identical criteria were followed in the collection of data for the tests executed with and without the tool. For each program unit: execution time, total number of executed segments and final coverage have been registered. The execution time was calculated excluding components not strictly dependent on test procedure as, e.g., compilation time or environment preparation.

The results collected up to this point do not allow us to draw significant statistical evaluations, as we could have expected given the problems i) and ii) above. We hope that adding many more results will allow us to overcome these problems. However, we can already derive some interesting conclusions.

- With regard to the use of BAT, we gained useful feedback to improve the tool interface and functionalities. More interestingly, we provided BAT with the option to suggest a set of paths that covers the branches as yet uncovered, which in practice provides a useful integration between BAT, that is static, with the dynamic coverage analyser.

- The experience with BAT confirmed the assumption that the paths derived by the tool are feasible with very good probability. All the paths suggested by the tool for the program units tested until now (cumulatively, about 60 paths for the BAT group) were feasible.

- The effectiveness of BAT is strongly affected by the tester's experience. In particular, we felt that a skilled tester can find BAT little useful, since he can derive the "right" paths immediately by his experience. In fact, an interesting observation was that, analysing the paths chosen by the expert tester for the program units in the STANDARD group, we saw, a posteriori, that he chose exactly the set of paths suggested by BAT. On the other hand, this can be considered a confirmation that BAT works fine.

A not expert tester, instead, can find the tool useful: the beginner felt that for the program units tested with BAT he could work in a more productive way and that he could derive a lower number of test cases to reach the customer requirements. Unfortunately, the data collected up to now do not allow us to make quantitative observations on this point. 


\subsection{Evaluation of the $\beta$ branch bound}

The $\beta$ branch bound, providing the number of complete paths needed to reach $100 \%$ branch coverage, was validated in the same test environment. The expected conclusion from the case study was that the bound is a good estimate of test effort to be used in project planning.

To this end, we have compared the theoretical (a priori) bound with the effective number of test cases used to test 55 program units. The comparison has been performed by considering both the absolute and the relative errors between the expected and the real number of test cases NT and by studying their empirical distribution. Specifically, the absolute error is given by:

$$
\Delta \beta_{\text {branch }}=\text { Bbranch }-\mathrm{NT}
$$

while the relative error by:

$$
\varepsilon \beta=\Delta \beta \text { branch } / \mathrm{NT}
$$

In order to evaluate the effectiveness of $\beta b r a n c h$, we considered the relative error. Of the 55 program units considered, only 1 observation gave a relative error greater than 1 . We thought that this only anomalous case could be discarded from the analysis without distorting the evaluation of results, since for all the other values the behaviour of the relative error was quite stable. From the data collected for the 54 program units analysed, we derived the histogram illustrated in figure 3 . We divided the interval $[-1,1]$ in which the relative error was contained into 20 sub-intervals of width 0.1 and reported them on the $\mathrm{X}$ axis. In the $\mathrm{Y}$ axis we reported the number of observations within each sub-interval. As shown in figure 4, we have also derived the empirical distribution of the relative errors; i.e., the $Y$ axis in figure 4, for each sub-interval, gives the ratio: (number of observed relative errors within the subinterval)/(total number of observations). This ratio is the classic estimate for the probability distribution of the relative errors (Lavenberg, 1983). After the first 35 observations, we observed that the estimate for the probability distribution had reached the steady state.

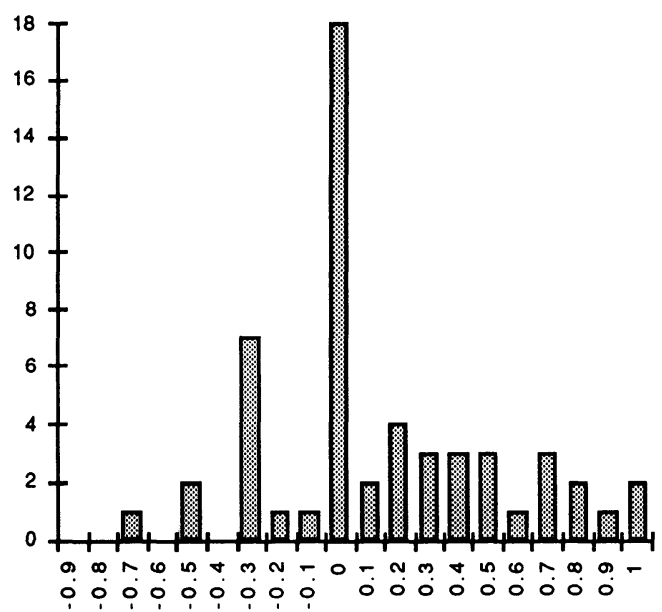

Figure 3 Observations of relative error $\varepsilon \beta$. 


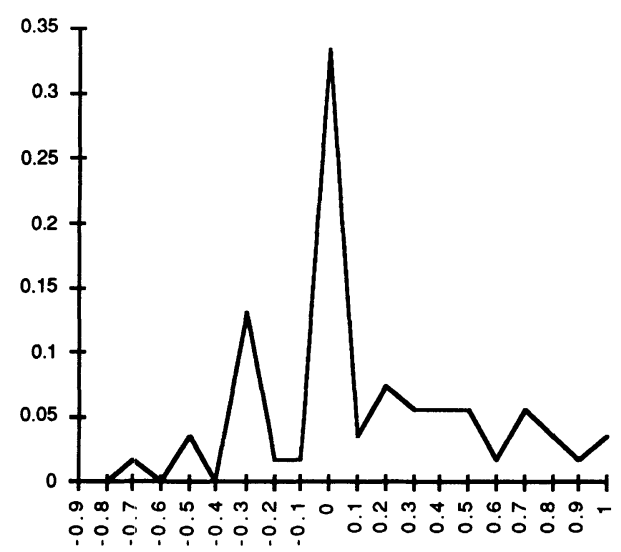

Figure 4 Empirical distribution of $\varepsilon \beta$.

We have computed the sample mean and variance for the $\mathrm{N}=54$ observations, which gave:

$$
\begin{aligned}
& \overline{\varepsilon_{\beta}}=\sum_{\mathrm{N}} \frac{\varepsilon_{\beta}}{\mathrm{N}}=0.12 \\
& \mathrm{~s}^{2}\left(\varepsilon_{\beta}\right)=\sum_{\mathrm{N}} \frac{\left(\varepsilon_{\beta}-\overline{\varepsilon_{\beta}}\right)^{2}}{\mathrm{~N}-1}=0.15
\end{aligned}
$$

The results confirm that the metric provides a good estimate for the test effort: 20 of the 54 observations (i.e., $37 \%$ ) gave a relative error less than $10 \%$. Let us observe that the $\beta$ branch predicts the number of test cases needed to reach a $100 \%$ branch coverage, while some of the tested program units reached a coverage from $80 \%$ up to $100 \%$. Presumably, if all the program units achieved full coverage, the observed values of $\varepsilon \beta$ should be even closer to 0 .

In order to generalise these results, we tried to identify a theoretical distribution underlying the empirical distribution. From figures 3 and 4 , we observed that the behaviour of $\varepsilon \beta$ seems similar to that of a theoretical normal distribution. To validate this hypothesis, i.e., to check that the differences between the observed results and those relative to a normal distribution with mean $\lambda=\overline{\varepsilon_{\beta}}$ and with standard deviation $\sigma=\sqrt{\mathrm{s}^{2}\left(\varepsilon_{\beta}\right)}$ are negligible, we applied the classical statistical tests $\chi^{2}$ and Kolmogorov-Smirnov (Knuth, 1981). Both tests confirmed that the hypothesis was acceptable with the $94 \%$ of the confidence level.

Finally, we have compared these results with the predictions that would have been obtained by using the cyclomatic complexity (McCabe, 1976) of the program ddgraph. It is defined as:

$$
v=|\mathrm{E}|-|\mathrm{V}|+2
$$

and is often used as a bound to the number of test cases needed to achieve branch coverage. In figure 5, the histogram obtained for $n$ is shown. We have considered the same 54 observation used to validate the branch bound (also for $v$ the observation that was anomalous for $\beta$ branch bound gave a very high value of the relative error and was discarded). Again, 20 of the 54 observations (37\%) gave a relative error less than $10 \%$. However, of the remaining 
34 observations, 8 laid outside the interval $[-1,1]$, and precisely between 1 and 2.67 . The sample mean and variance gave respectively: 0.37 and 0.39 . So, we can conclude that $v$ is not as reliable as $\beta$ branch to estimate the test cases needed to achieve branch coverage. Besides, the empirical distribution for the relative error could not be approximated by a theoretical normal distribution as we have observed by applying the statistical test $\chi^{2}$.

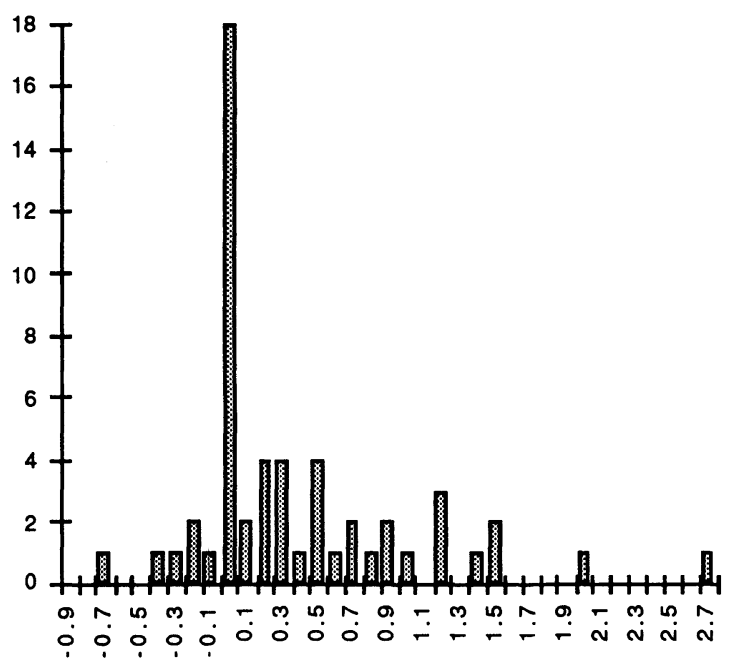

Figure 5 Observations of relative error $\varepsilon_{v}$.

\section{CONCLUSIONS}

We have described a real world case study aimed at evaluating a) the usefulness of a prototype tool that automatically derives a set of paths covering every branch in a program unit and $b$ ) the effectiveness of the $\beta$ branch bound in predicting the number of test cases needed to achieve $100 \%$ branch coverage.

For the first point we could only provide some preliminary evidence that the tool is actually useful, though only for not expert testers. Improving the automation of the basic test phase, however, was commonly felt as a positive innovation, since the testers find this phase quite tedious.

We are continuing the experimentation and we hope to be able to confirm the usefulness of the BAT tool in a more objective way. Anyway, the experience gained with the usage of the tool allowed us to make useful improvements to the tool interface and functionalities. Besides, we could confirm the effectiveness of the method used by the tool to generate the test paths, since all the paths suggested were feasible.

For the second point, the observed results confirmed that the $\beta$ branch bound provides a good estimate for the test effort: 20 of the 54 observations (37\%) gave a relative error less than $10 \%$. By applying classical statistical tests, we could also conclude that the empirical distribution of the relative error between the expected number of test cases and the observed 
number can be considered a normal distribution with $\lambda=0.12$ and $\sigma^{2}=0.15$ with the $94 \%$ of the confidence level.

\section{ACKNOWLEDGEMENTS}

The authors thank Andrea Baldanzi, Giorgio Morini and Giovanni My for their helpful support in the experimentation with the BAT tool.

They also wish to express their gratitude to Dr. Massimo De Sanctis, head of the Software Development Department of Ericsson Telecomunicazioni S.p.A., for his prompt availability to provide the test environment for the case study described in the paper.

\section{REFERENCES}

Beizer, B., Software Testing Techniques, Second Edition, Van Nostrand Reinhold, New York, 1990.

Bertolino, A., Unconstrained Edges and Their Application to Branch Analysis and Testing of Programs, Journal of Systems and Software, 20 (2), 125-133 (1993).

Bertolino, A. and Marrè, M., Automatic Generation of Path Covers Based on the Control Flow Analysis of Computer Programs, IEEE Trans. on Software Engineering, 20(12), 885899 (1994).

Bertolino, A. and Marrè, M., How many paths are needed for branch testing?, to appear onThe Journal of Systems and Software (1995).

Lavenberg, S.S., Computer Performance Modelling Handbook, Academic Press, New York, 1983.

McCabe, T. J., A Complexity Measure, IEEE Trans. on Software Engineering, SE 2 (4), 308320 (1976).

Hecht, M. S., Flow Analysis of Computer Programs, Elsevier, New York, 1977.

Jacobson, I., et al., Object Oriented Software Engineering, Addison-Wesley, 1992.

Knuth, E. D., The Art of Computer Programming, Vol. 2, Addison-Wesley, Reading, Massachusetts, 1981.

Rapps, S. and Weyuker, E. J., Selecting Software Test Data Using Data Flow Information, IEEE Trans. on Software Engineering, SE-11 (4), 367-375 (1985).

STW, Software TestWorks, Test Coverage Tools: TCAT, S-TCAT, TCAT-PATH, TSCOPE., SR Software Research, Inc., San Francisco, 1991.

Yates, D. F. and Malevris, N., Reducing the Effects of Infeasible Paths in Branch Testing, ACM SIGSOFT Software Engineering Notes, 14 (8), 48-54 (1989).

Antonia Bertolino graduated cum laude in Electronic Engineering at the University of Pisa. Since 1986 she has been a researcher of the Italian National Research Council (CNR) in Pisa. Her research interests are in testing theory and techniques and in testing automation. She is an Associate Editor of the Journal of Systems and Software.

Raffaela Mirandola received the Laurea degree in Computer Science from the University of Pisa and Ph.D. degree in Computer Science from the University of Rome Tor Vergata. Her main research interests are in the areas of modelling, validation and performance evaluation of computer and communication systems.

Emilia Peciola graduated in Computer Science at the University of Pisa. She has been working in Ericsson Telecomunnication since 1985, involved in software development for telecommunication systems. Since 1993 she has been working in R\&D as section manager of Object Oriented software developed for new telecommunication systems. 